\title{
OBSERVATIONAL LIMITS ON TERRESTRIAL-SIZED INNER PLANETS AROUND THE CM DRACONIS SYSTEM USING THE PHOTOMETRIC TRANSIT METHOD WITH A MATCHED-FILTER ALGORITHM
}

\author{
Laurance R. Doyle, ${ }^{1}$ Hans J. Deeg, ${ }^{2,3}$ Valerij P. Kozhevnikov, ${ }^{4}$ Brian Oetiker, ${ }^{5}$ Eduardo L. Martín, ${ }^{2}$ \\ J. Ellen Blue, ${ }^{6}$ Lee Rottler, ${ }^{7}$ Remington P. S. Stone, ${ }^{8}$ Zoran Ninkov, ${ }^{9}$ Jon M. Jenkins,,${ }^{10}$ \\ Jean Schneider, ${ }^{11}$ Edward W. Dunham, ${ }^{12}$ Moira F. Doyle, ${ }^{13}$ and Efthimious Paleologou ${ }^{14}$ \\ Received 1999 August 18; accepted 1999 December 28
}

\begin{abstract}
A light curve of the eclipsing binary CM Draconis has been analyzed for the presence of transits of planets of size $\geq 2.5$ Earth radii $\left(R_{\mathrm{E}}\right)$, with periods of 60 days or less, and in coplanar orbits around the binary system. About 400 million model light curves, representing transits from planets with periods ranging from 7 to 60 days, have been matched/correlated against these data. This process we call the "transit detection algorithm" or TDA. The resulting "transit statistics" for each planet candidate allow the quantification of detection probabilities, and of false-alarm rates.

Our current light curve of CM Dra has a coverage of $1014 \mathrm{hr}$ with 26,043 individual points, at a photometric precision between $0.2 \%$ and $0.7 \%$. Planets significantly larger than $3 R_{\mathrm{E}}$ would constitute a "supranoise" detection, and for periods of 60 days or less, they would have been detected with a probability greater than $90 \%$. "Subnoise" detections of smaller planets are more constrained. For example, $2.5 R_{\mathrm{E}}$ planets with 10 day periods or less would have been detected with an $80 \%$ probability. The necessity for predicted observations is illustrated with the nine top planet candidates that emerged from our TDA analysis. They are the planet candidates with the highest transit statistics from the 1994-1998 observing seasons, and for them transits for the 1999 observing season were predicted. Of the seven candidates that were then observationally tested in 1999, all were ruled out except one, which needs further observational confirmation. We conclude that the photometric transit method is a viable way to search for relatively small, inner extrasolar planets with moderate-sized telescopes using CCD photometry with a matching-filter analysis.

Subject headings: binaries: eclipsing - methods: statistical - planetary systems stars: individual (CM Draconis) - techniques: photometric
\end{abstract}

\section{INTRODUCTION}

The idea that extrasolar planets may be detected by transits across the disk of stars goes back to a suggestion by Struve (1951) with subsequent full development by Rosenblatt (1971) and Borucki \& Summers (1984) (see also Hale \& Doyle 1994; Deeg 1998). Schneider \& Chevreton (1990) first suggested that eclipsing binaries would be good candidates for such a search, based on their orbital planes

\footnotetext{
${ }^{1}$ Search for Extraterrestrial Intelligence Institute, 2035 Landings Drive, Mountain View, CA 94043; 1doyle@seti.org.

${ }^{2}$ Instituto de Astrofísica de Canarias, E-38200 La Laguna, Tenerife, Spain; hdeeg@bigfoot.com.

${ }^{3}$ Centro de Astrobiología, E-28807 Torrejon de Ardoz, Madrid, Spain.

${ }^{4}$ Astronomical Observatory, Ural State University, Lenin Avenue 51, 620083 Ekaterinburg, Russia.

${ }^{5}$ University of New Mexico, Department of Astronomy and Physics, Albuquerque, NM 87131.

${ }^{6}$ SRI International, 333 Ravenswood Avenue, Menlo Park, CA 94025.

7 University of California, Lick Observatory, 1156 High Street, Santa Cruz, CA 95060.

${ }^{8}$ University of California, Lick Observatory, Mount Hamilton, CA 95040 .

${ }^{9}$ Center for Imaging Science, Rochester Institute of Technology, Rochester, NY 14623.

${ }^{10}$ Search for Extraterrestrial Intelligence Institute, MS 245-3, NASA Ames Research Center, Moffett Field, CA 94035.

${ }^{11}$ CNRS-Observatoire de Paris, 92195 Meudon, France.

12 Lowell Observatory, Mars Road, Flagstaff, AZ 87000.

${ }^{13}$ FIDM, 1212 Raintree Circle, Culver City, CA 90230.

${ }^{14}$ University of Crete, Skinakas Observatory, Post Office Box 1527, 71110 Iraklion, Greece.
}

already being nearly edge-on. There are a number of advantages to choosing small eclipsing binaries for such searches. These include the obvious advantage that eclipsing binary systems are already known to have their orbital planes very nearly edge-on to the observer's line of sight, with subsequent planet formation expected to take place near the same plane. An additional damping of planetary orbits into the binary plane due to precession could also add to their coplanarity (Schneider 1994; Schneider \& Doyle 1995).

For detection of terrestrial-sized planets, smaller stellar systems are preferred, as they exhibit greater brightness variations during the transit of a given sized planet. The stellar components of the CM Dra binary (A and B) have a total disk area of about $12 \%$ that of the solar disk, allowing an order-of-magnitude improvement in detectability. The luminosity of the two components is only $1.03 \%$ of the solar luminosity; they have a separation of 3.76 solar radii (about 14.9 times the radius of CM Dra A; Lacy 1977), and a mutual orbital period of 1.268389861 days (Deeg et al. 1998). Consequently, the nearest stable third body would have a period of somewhat less than 7 days (Holman \& Wiegert 1999 and references therein), with planets of periods up to about 35 days receiving the same energy from CM Dra A\&B as the terrestrial planets receive from the Sun.

As a photometric comparison, a Neptune-sized planet in transit across one of the components of the CM Dra system would cause an $0.8 \%$ drop in brightness, while an Earthsized planet would cause an $0.07 \%$ decrease in brightness. 
Ground-based $1 \mathrm{~m}$ photometric precision may be limited to about $0.1 \%$ (Young et al. 1991), but improvement up to $0.015 \%$ photometry with $4 \mathrm{~m}$ class telescopes - mainly from reduced atmospheric scintillation noise - can be expected (Gilliland \& Brown 1992).

It should also be noted that planetary transit events will occur across the disks of close eclipsing binaries in a quasiperiodic manner, as the phase of the binary components will be different between subsequent planetary transits (see Fig. 1 in Deeg et al. 1998). Because these are quasi-periodic photometric attenuations, they can provide unique signatures of transit events, ruling out attenuations due to starspots on the stellar surface, for example. Of most importance, such transit attenuations have a significantly shorter duration, on average, than single star transit events (50 minutes compared with several hours) and thereby show a sufficiently different power spectrum from major sources of observational variability, such as nightly extinction variations (Deeg et al. 1998). However, detections do not take place in frequency space, as the power of occasional transits is minuscule compared to the total observational noise power. Thus the observed light curve needs to be "matched" (correlated) in the time regime against synthetic light curves of all possible transit models that could occur across the observational range of period-phase space (see Brandmeier \& Doyle 1996; Jenkins, Doyle, \& Cullers 1996; Deeg et al. 1998).

As an aside, a further advantage of small eclipsing binaries as targets for photometric planet searches is that outer Jovian mass planets around such systems may also be detected without transits - using the same photometric data - by a precise timing of eclipse minima. As is well known, the mutual binary eclipse minima constitute a periodic signal in themselves whose periodic variations in time can be indicative of drifts in the position of the binary system toward or away from the observer due to a third mass in orbit around the binary system. The subsequent light travel time difference across the binary/third-mass barycenter produces the periodic variation in the time of eclipses (see, for example, Hertz, Wood, \& Cominsky 1995; Doyle et al. 1998). An analysis constraining the presence of outer Jovian mass planets with periods shorter than about 6 years around the CM Dra system is being published separately (Deeg et al. 2000).

The remainder of this paper will outline the observational coverage of CM Dra obtained by the Transit of Extrasolar Planets (TEP) network; focus on a detailed description of the transit detection algorithm (TDA), with its application to the search for planets of sizes $\gtrsim 2.5 R_{\mathrm{E}}$ around CM Dra with periods from 7 to 60 days; and finally discuss the resultant planetary candidates and their observational dismissal or confirmation.

\section{OBSERVATIONAL COVERAGE OF CM DRA}

A detailed description of observations of CM Dra taken in the years 1994-1996, along with a list of the observatories, detectors, data reduction procedures, and software is given in Deeg et al. (1998; hereafter TEP1). Previous accounts of the TEP network are also given by Schneider \& Doyle (1995), Doyle et al. (1996), and Deeg et al. (1997).

Our total high-precision observations of CM Dra taken from 1994 through 1998 are included in the analysis presented here. Observations in 1999 were performed to confirm or rule out specific predicted planetary transit can- didates that resulted from the TDA general analysis, and results of these observations will be presented in $\S 6$. In addition to the data presented in TEP1, 250 hours of highprecision observational coverage were obtained in 1997, 106 hours in 1998, and 41 hours in 1999. These more recent observations were performed at the Crossley $(0.9 \mathrm{~m})$ telescope at Lick Observatory, at the IAC80 (0.8 m) and OGS $(1 \mathrm{~m})$ telescopes of the Instituto de Astrofísica de Canarias, the Kourovka $0.7 \mathrm{~m}$ telescope of the Ural State University in Ekaterinburg, Russia, and at the Capilla Peak $0.6 \mathrm{~m}$ of the University of New Mexico. A summary of all TEP observations of sufficient photometric precision is given in Table 1. The total light curve, to date, consists of 26,042 data points with a photometric precision (standard deviation over the mean differential brightness of CM Dra) between $0.2 \%$ and $0.7 \%$, giving $1014 \mathrm{hr}$ of total coverage of CM Dra. However, only the 1994-1998 observations24,874 data points covering 973 hours - were used in the TDA search for planetary candidates. The specific aperture photometry software VAPHOT, developed for this project, is presented in Deeg \& Doyle (2000).

Within the 973 hour (1994-1998) light curve, many possible transit events could have occurred. This can be characterized by the number of transits, $N_{\mathrm{tr}}$, that would have been observed, if a planet with a particular period had been present. The value $N_{\text {tr }}$ takes into account that a planet orbiting an eclipsing binary normally causes two transit events per period (see Fig. 1 in TEP1). The probabilities that a planet with random epoch and period (within certain period ranges) causes a specific number of transits in the observed light curve we will call the "observational probability" $p_{o}\left(N_{\mathrm{tr}}\right)$ (see Fig. 1). In addition to being in the observational data, however, the transit events have to give a sufficiently strong signal (i.e., total sum of transit event depths compared to the background noise level) for a given candidate to be detectable. The probability of a given transit signal being detectable above the noise, which we will call the "intrinsic detection probability," $p_{i}$, will be given in $\S 4$, following standard detection theory. The overall probability of detection of a given planetary transit candidate, $p_{d}$, then is

$$
p_{d}=p_{o} p_{i}
$$

Since a single transit from a large planet (significantly larger than $3 R_{\mathrm{E}}$ ) would have been fairly obvious in the light curve (and therefore has $p_{i}=1$ ), its probability of detection is $p_{d}=p_{o}\left(N_{\mathrm{tr}, \min }=1\right)$, where $p_{o}\left(N_{\mathrm{tr}, \min }\right)$ is the probability that at least $N_{\mathrm{tr}, \min }$ transits are in the light curve (Fig. 1b). For planets larger than $3 R_{\mathrm{E}}$, then, from the data of Figure $1 b$, we have $p_{o} \approx 90 \%$ for planetary periods of 60 days, with $p_{o}$ being significantly greater for shorter period $\left(p_{o} \approx 98 \%\right.$ for periods of 25 days, for example). Having observed no such transit events, we can thus state, with a confidence of better than $90 \%$, that there are no planets significantly larger than $3 R_{\mathrm{E}}$ in coplanar orbits of 60 days or less around the $\mathrm{CM}$ Dra system. It should be noted that - for the derivation of a unique period and epoch of a planet, by transit measurements alone - at least three transit events need to be identified in the light curve. Also, in $\S 4$, it will be shown that there is a close relationship between the number of transits, $N_{\mathrm{tr}}$, of a particular candidate and its intrinsic detection probability $p_{i}$.

For an estimation of the effect of additional observations, or of the effect of taking subsamples from the observed data 
TABLE 1

TEP NETWORK OBSERVATIONS OF CM DRACONIS (hours)

\begin{tabular}{cccccccr}
\hline \hline Observatory & 1994 & 1995 & 1996 & 1997 & 1998 & 1999 & Total \\
\hline Crossley $(0.9 \mathrm{~m}) \ldots \ldots \ldots$ & 65 & 50 & 46 & 21 & 72 & 41 & 295 \\
IAC $(0.8 \mathrm{~m}) \ldots \ldots \ldots \ldots$ & 38 & 62 & 22 & 43 & 20 & $\ldots$ & 185 \\
Kourovka $(0.7 \mathrm{~m}) \ldots \ldots$. & $\ldots$ & $\ldots$ & 68 & 87 & $\ldots$ & $\ldots$ & 155 \\
Capilla $(0.6 \mathrm{~m}) \ldots \ldots \ldots$ & $\ldots$ & $\ldots$ & 18 & 99 & $\ldots$ & $\ldots$ & 117 \\
OHP $(1.2 \mathrm{~m}) \ldots \ldots \ldots \ldots$ & 11 & 34 & 22 & $\ldots$ & $\ldots$ & $\ldots$ & 67 \\
Mees $(0.6 \mathrm{~m}) \ldots \ldots \ldots \ldots$ & 53 & $\ldots$ & $\ldots$ & $\ldots$ & $\ldots$ & $\ldots$ & 53 \\
INT $(2.5 \mathrm{~m}) \ldots \ldots \ldots \ldots$ & $\ldots$ & $\ldots$ & 42 & $\ldots$ & $\ldots$ & $\ldots$ & 42 \\
JKT $(1.0 \mathrm{~m}) \ldots \ldots \ldots \ldots$ & $\ldots$ & 39 & $\ldots$ & $\ldots$ & $\ldots$ & $\ldots$ & 39 \\
WISE $(1.0 \mathrm{~m}) \ldots \ldots \ldots \ldots$ & $\ldots$ & $\ldots$ & 28 & $\ldots$ & $\ldots$ & $\ldots$ & 28 \\
Skinakas $(1.3 \mathrm{~m}) \ldots \ldots \ldots$ & 19 & $\ldots$ & $\ldots$ & $\ldots$ & $\ldots$ & $\ldots$ & 19 \\
OGS $(1.0 \mathrm{~m}) \ldots \ldots \ldots \ldots$ & $\ldots$ & $\ldots$ & $\ldots$ & $\ldots$ & 14 & $\ldots$ & 14 \\
Total $\ldots \ldots \ldots \ldots \ldots \ldots$. & 186 & 185 & 246 & 250 & 106 & 41 & 1014 \\
\hline
\end{tabular}

NotE. - Coverage is defined as continuous observations without any interruption lasting more than 15 minutes.

(such as a subsample consisting only of the highest precision photometry) on the transit coverage, the following relationship between $p_{o}, N_{\mathrm{tr}}$, and $T$ (time of coverage) can be derived:

$$
p_{o}\left(N_{\mathrm{tr}, 1}, T_{1}\right) \approx(1 / k) p_{o}\left(N_{\mathrm{tr}, 0}, T_{0}\right),
$$

where $k=T_{1} / T_{0}$ and $N_{\mathrm{tr}, 1} \approx k N_{\mathrm{tr}, 0}$ (of course, $N_{\mathrm{tr}}$ can only take integer values). A doubling of the observing time therefore approximately doubles the number of observed transits, as might be expected.

\section{THE PHOTOMETRIC TRANSIT DETECTION ALGORITHM: A MATCHED-FILTER APPROACH}

In this section we outline a procedure for the detection of planetary transit signals near the photometric noise in the light curves of eclipsing binaries. Although the application in this paper is specific to the CM Dra system, this method is generally applicable to the detection of such signals in any light curves from eclipsing binaries or even single stars.

The procedure for instituting the TDA is as follows. First, mutual eclipses of CM Dra are removed from the observed
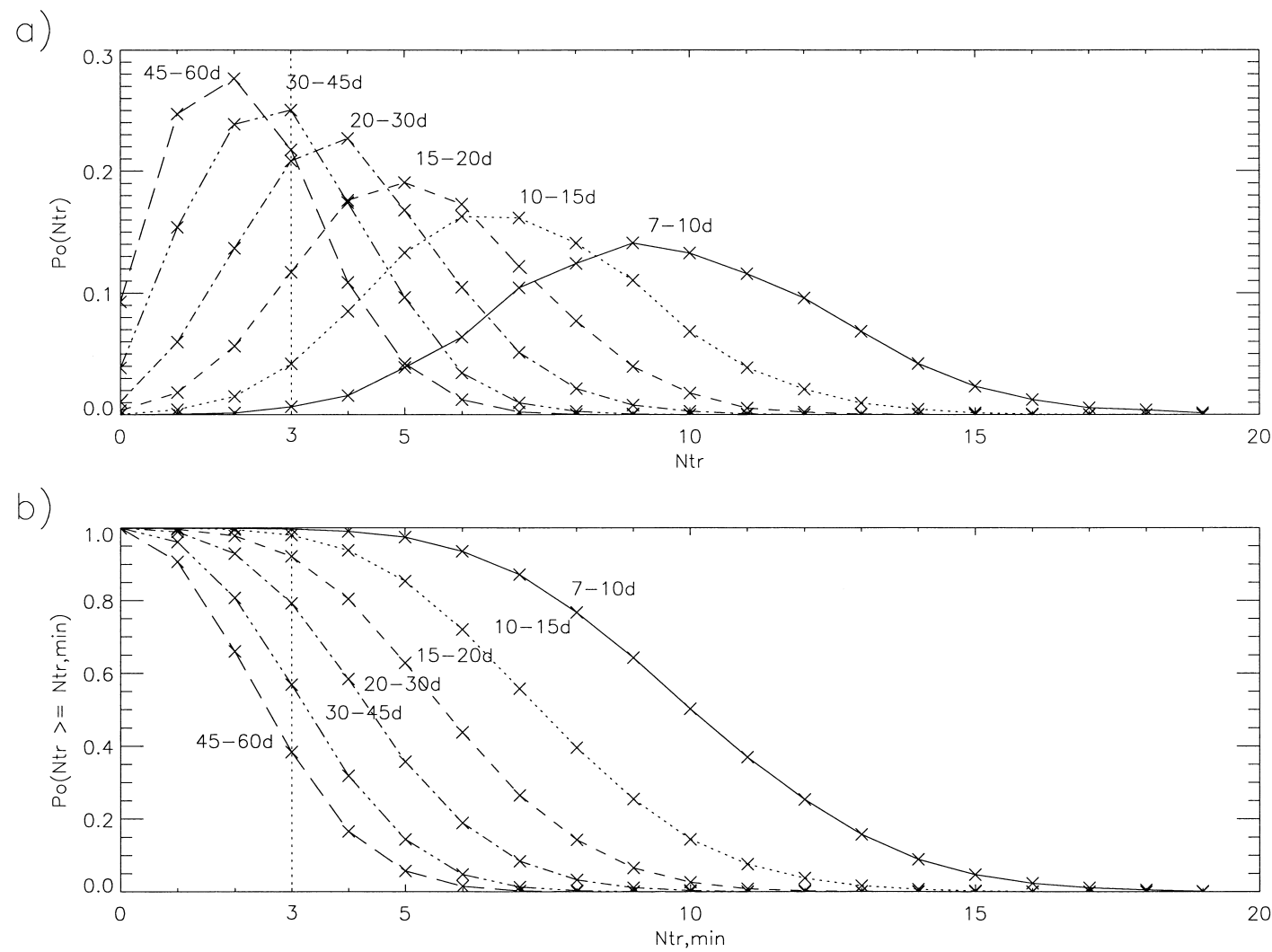

FIG. 1.- (a) Probability $p_{o}\left(N_{\mathrm{tr}}\right)$, that just $N_{\mathrm{tr}}$ transits from a planet are in our observed light curve. (After a certain amount of coverage, for example, the probability of having only one transit goes down, etc.) Curves are labeled with the period range of the planets. The probabilities $p_{o}$ were derived from the insertion of 60,000 planets with random periods and epochs into our data. (b) Cumulative probability $p_{o}\left(N_{\mathrm{tr}} \geq N_{\mathrm{tr}, \min }\right)$, that $N_{\mathrm{tr}, \min }$ or more transits from a planet are in our observed light curve. For example, for planets with periods between 20 and 30 days, the probability that there are three or more transits in our data is about 0.79 , whereas for planets with periods of less than 10 days this probability is better than 0.99 . 
light curve by the subtraction of model eclipses. ${ }^{15}$ The light curve is then converted to relative flux values, $D\left(t_{i}\right)=$ $\Delta F / F_{0}$, where the zero point, $F_{0}$, is the average off-eclipse brightness of (in this case) CM Dra, and $\Delta F$ is the difference in the flux from CM Dra and the flux from the nonvariable comparison stars in the field (TEP1). However, some residuals from nightly extinction variations ${ }^{16}$ are still contained in $D\left(t_{i}\right)$.

For a transiting test planet with radius $R$, period $P$, and epoch $^{17} E$, a model transit light curve $M_{p}\left(t_{i}, R, P, E\right)$ is generated that describes the relative brightness variations of the star in the presence of a test planet, at all times $t_{i}$, where observational data have been taken (Fig. 2 below). It should be noted that $M_{p}\left(t_{i}, R, P, E\right)=0$ except for the set of times, $t_{\mathrm{tr}}$, when a transit occurs. Due to computing constraints, circular orbits are assumed in all planet models, and orbital effects due to tidal precession (as well as noncentral potentials or general relativity) are also not presently included.

Two fits are now performed on light-curve data $D\left(t_{i}\right)$, which led to a comparison of the cases "no-transit" and "transit present from model $M_{p}$ ":

\footnotetext{
${ }^{15}$ The model eclipse was obtained from the averaging of several tens of observed eclipses; see TEP1.

${ }^{16}$ Although first-order extinction was removed in the initial processing of the data (see TEP1), higher order effects from the color difference between CM Dra and the comparison stars can remain.

${ }^{17}$ The epoch $E$ is defined as the time when a candidate planet crosses in front of the binary's barycenter across the observer's line of sight.
}

1. The no-transit, extinction-only case is described by a parabolic ${ }^{18}$ fit, $f_{e}\left(t_{i}\right)$ to each night's section of the light curve $D\left(t_{i}\right)$.

2. The "transit present" case is described by a parabolic fit, $f_{p}\left(t_{i}, R, P, E\right)$, which is a fit to each night's block of $\left[D\left(t_{i}\right)-M_{p}\left(t_{i}, R, P, E\right)\right]$, the difference between the data and the model transits.

Of course, $f_{e}$ and $f_{p}$ are different only on nights with possible transits, i.e., nights which contain times $t_{i}=t_{\mathrm{tr}}$, where $M_{p}\left(t_{i}, R, P, E\right) \neq 0$. These model fits, and the subsequent calculations, need therefore only be performed for such nights.

We then evaluate which of these two fits describes the data better by comparing residuals for the extinction-only light curve,

$$
r_{e}\left(t_{i}\right)=\left|D\left(t_{i}\right)-f_{e}\right|,
$$

with the residuals for the planet-being-present case,

$$
r_{p}\left(t_{i}, R, P, E\right)=\left|\left(D\left(t_{i}\right)-M_{p}\left(t_{i}, R, P, E\right)\right)-f_{p}\right| .
$$

This is performed through the calculation of a transit statistic $\kappa$ :

$$
\begin{aligned}
& \kappa\left(t_{i}, R, P, E\right)=\left[r_{e}\left(t_{i}\right)-r_{p}\left(t_{i}\right)\right]\left[t_{i+1}-t_{i}\right] / \sigma_{D} \\
& \text { if } t_{i+1}-t_{i} \leq 10 \mathrm{~min} \text {, } \\
& \kappa\left(t_{i}, R, P, E\right)=0 \quad \text { if } t_{i+1}-t_{i}>10 \mathrm{~min} \text {, }
\end{aligned}
$$

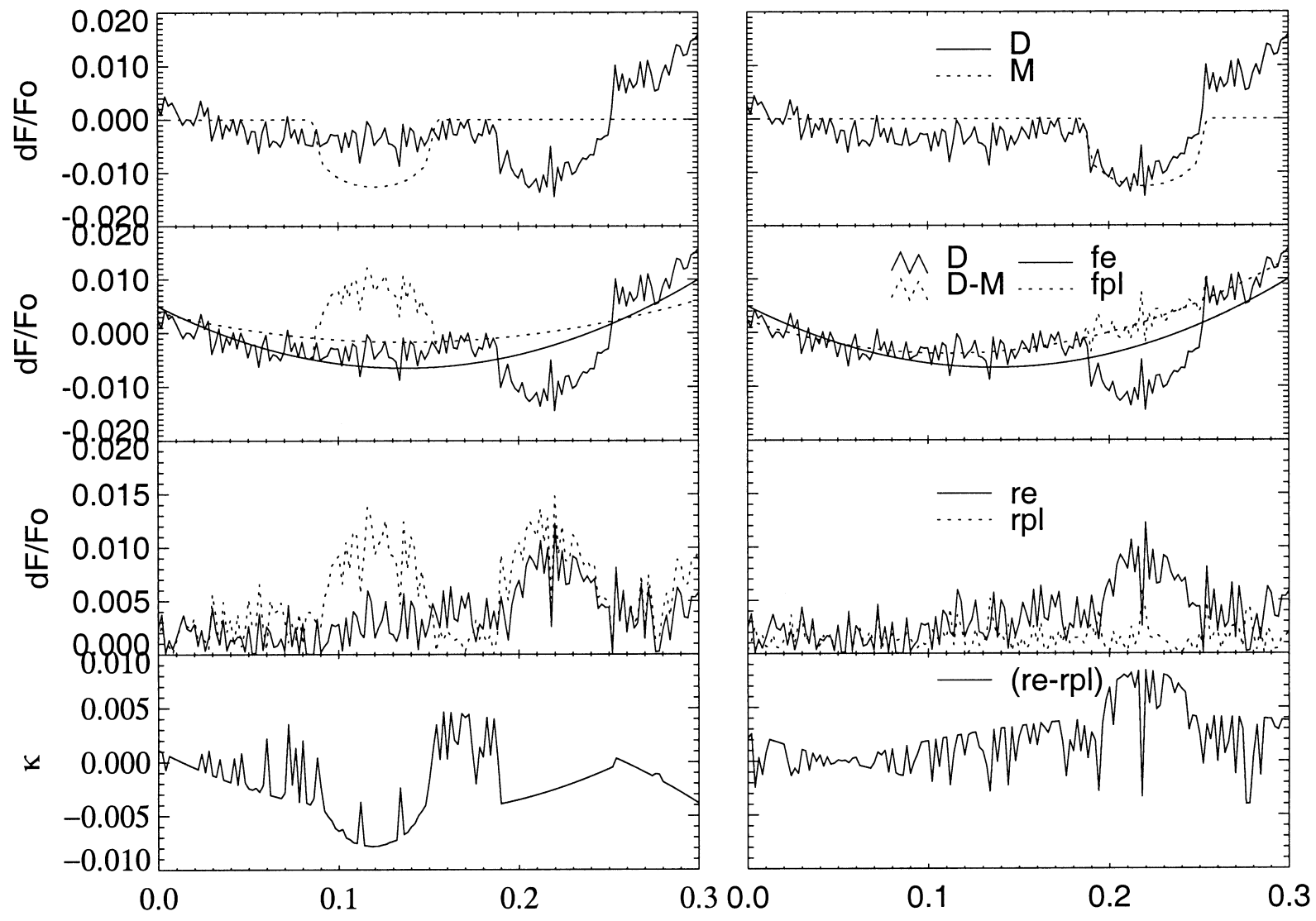

FIG. 2.-Derivation of the coefficient $\kappa\left(t_{i}\right)$. The uppermost panels show a nightly light curve $D$ (solid line) and a test model $M_{p}$ (dashed line). In panels on the left, $M_{p}$ aligns with a range of inconspicuous data, whereas in the right-hand panels, $M_{p}$ lines up with a possible transit in the data. The second row of panels shows again the light curve $D$ (solid jagged line) and the subtraction of the model, $D-M_{p}$ (dashed jagged line). Overlaid are $f_{e}$ (the fit to $D ;$ smooth solid line) and $f_{p}$ (the fit to $D-M_{p}$; dashed smooth line). The third row of panels shows the residuals $r_{e}$ (solid line) and $r_{p}$ (dashed line). In the bottom panels the difference of the residuals, $\kappa=r_{e}-r_{p}$, is plotted. It can be seen that in the "planet-absent" case (left panels) $\kappa\left(t_{\mathrm{tr}}\right)<0$, and in the "planet-present" case $($ right panels) $\kappa\left(t_{\mathrm{tr}}\right)>0$ at the times where $r_{p}<r_{e}$. 
where $\sigma_{D}$ is the rms of one night's observational data $D\left(t_{i}\right)$. The distinction between equation (5a) and equation (5b) assures that "holes" in the light curve of more than 10 minutes duration are ignored. The scaling by $\left(t_{i+1}-t_{i}\right)$ in equation (5a) was needed to account for the various time increments that appear in sections of the light curve originating from different telescopes (see Table 2 in TEP1 for exposure times and duty cycles of the different telescopes). If equation (5a) applies, then

$$
\kappa\left(t_{i}\right)<0 \text { when } r_{e}\left(t_{i}\right)<r_{p}\left(t_{i}\right) .
$$

This is the normal case, when no transit is apparent in $D\left(t_{i}\right)$, and $f_{e}$ fits better than $f_{p}$. The other case is

$$
\kappa\left(t_{i}\right)>0 \text { when } r_{e}\left(t_{i}\right)>r_{p}\left(t_{i}\right) .
$$

In this case, $f_{p}$ is a better description of the data than $f_{e}$, which means that the data could contain a transit event at time $t_{i}$.

In Figure 2 we show the derivation of the transit statistic in the cases of a poor (left panel) and a good (right panel) transit model fit to the light-curve data. One can see that the poor fit will result in negative values of the transit statistic while a good fit will give positive values; the higher the value of the transit statistic, the better the candidate model fit.

The final "complete transit statistic" $C$ of a given planet model transit is then obtained by a summation of the $\kappa\left(t_{i}\right)$ of all points of the light curve (though only nights with transits of the model planet have to be considered) with

$$
C(R, P, E)=\sum_{i=1}^{n} \kappa\left(t_{i}\right) .
$$

The complete transit statistic, $C$, is hence a normalized (by the rms of each night) indication of the difference in area under the light curve (units: $\Delta F / F_{0} \times$ time) between the "transit"-present and no-" transit" fits. This linear scaling in the difference of the areas was preferred over a quadratic $\left(\chi^{2}\right)$ one because of the lower weighting that is given to outliers. This procedure to calculate $C(R, P, E)$ is also insensitive to brightness variations with frequencies of $\gtrsim 4$ hours, such as might result from differential extinction or from partial phases of starspot rotation. It is also insensitive to the setting of the zero point for the light-curve data. This is an important point for evaluations of transits that last a large fraction of an observing night, i.e., where a zero point of the light curve cannot be reliably set. ${ }^{19}$ Very long transit events - i.e., ones that begin before the start of a night's observations and finish after the end of observations (these occur if the planet transit occurs simultaneously with a binary eclipse; see Fig. 1 in TEP1) - will not, however, be included in this detection approach. ${ }^{20}$ For detached systems, such long transit events are much rarer than the shorter transits, which are about 50 minutes in duration. By

\footnotetext{
${ }^{19}$ It was found that an absolute setting of the zero point by keeping a fixed set of reference stars through all nights is not sufficiently precise because of nightly atmospheric variations and because of the use of various telescopes with slightly different wavelength responses (filter sets, CCDs, etc). The light curves' zero point was therefore set only approximately in the initial photometric reductions described in TEP1 and is then left unconstrained for the fitting routines described here.

${ }^{20}$ The eclipse minima during transits may, however, be compared for any attenuations with the out-of-transit primary or secondary eclipse minima depth. Any transit events during binary eclipses will, of course, give twice the signal as the projected "area" of the star is halved.
}

excluding long transits, then, we are taking a conservative detection limit approach. An advantage of this method is, however, that $C(R, P, E)$ is sensitive to the best match of the shape of an observed light-curve event to a given model transit, as well.

Keeping the test planets' radius $R$ constant, values for the transit coefficients are then calculated scanning through a grid of values of $E$ and $P$, with a two-dimensional array of values $C(E, P)$ as the result. Exploratory scans across small sections of the $(E, P)$ parameter space were performed for planetary candidate radii of $R=2.0,2.5,3.0$, and $4.0 R_{\mathrm{E}}$, in order to evaluate the detection statistics. The only complete scans through the entire parameter space were then performed for periods from 7 to 60 days, for a planetary radius of $R=2.5 R_{\mathrm{E}}$. (The justification for choosing $R=2.5 R_{\mathrm{E}}$ is given in $\S 4$ on detection statistics.)

The possible planets' epochs, $E$, are searched completely, if $C(E, P)$ is calculated between an arbitrary initial epoch $E_{0}$, and $E_{0}+P$ (we used $E_{0}=$ JD 2,450,000.0). In order not to miss any transits, the step sizes for the epoch and the period must be (after Jenkins et al. 1996)

$$
\Delta E=t_{d} f_{\mathrm{ov}}
$$

and

$$
\Delta P=P t_{d} f_{\mathrm{ov}} / t_{j},
$$

where $t_{d}$ is the typical duration of a short transit (we used 0.02777 days $=40$ minutes), $f_{\text {ov }}$ is an overlap factor between adjacent scans (we used $f_{\mathrm{ov}}=0.5$ ), and $t_{j}$ is the time difference between the first and the last point in the light curve. For the light curve from observations between 1994 and 1998 , with $t_{j}=1526$ days, about $4 \times 10^{8}$ values of $C$ have to be calculated to completely scan for all distinguishable transit models from planets with periods from 7 to 60 days. Considering that each value of $C$ is the result of the summation in equation (8), it is obvious that the problem is computationally very intensive, and would have normally required about 2 years of CPU time on a current high-performance workstation.

To reduce the computational load, we divided the light curve into five yearly sections, each of which covered a time range of less than 200 days (CM Dra was observed only within the seven months of March through September in each year). Thus, without missing any transits within the same year, $\Delta P$ could be incremented with larger step sizes, based on each year's $t_{j}=200$ days (eq. [10]). Therefore, the length of the yearly light curve that had to be considered, i.e., the number of points in the summation equation (8), was decreased to about a fifth of the previous number. However, there were now five scans made, one for each year, which created five arrays $C(E, P)$ with identical dimensions. The total saving in computing time was thus $1526 /$ 200 , or a reduction factor of about 7.6, making it possible to perform the complete calculation in about 2 months using two workstations, with an optimized code that allowed us to perform about 30 planet-model evaluations per second. These five scans covered the period range with 30,917 steps at period increments between $4.2 \times 10^{-3}$ and $4.8 \times 10^{-3}$ days and covered the epoch with increments of 0.014 days, for a total of $5.6 \times 10^{7}$ planet model scans being performed altogether. An additional advantage of this partitioning of the light curve is that it became possible to add in data from future observations so that only the additional data would need to be evaluated. 
However, such savings in computational time did not come without some cost. Consider a planet with an intrinsic epoch and period, $\left(E_{p}, P_{p}\right)$, that has caused, for example, four transits, two of which were observed in 1994 and two of which were observed in 1997. In order not to miss the "alignment" between all four of them, the scans would have to be performed with the step sizes given by equations (9) and (10), (based on $t_{j}=1526$ days). The scans for the individual years, with $t_{j}=200$ days, are only sufficiently fine to find any alignment between transits within that same year. Thus, in 1994, there will be a local maximum, $C_{94}\left(E_{j}, P_{k}\right)$, (where $j$ and $k$ are the indices in the array spanning $E$ and $P)$. The position of this maximum at $\left(E_{j}, P_{k}\right)$ will be within $\Delta E / 2$ and $\Delta P / 2$ of the intrinsic values $E_{p}$ and $P_{p}$. The scan for the year 1997 will now also find a maximum, $C_{97}$, which is also constrained to be within $\Delta E / 2$ and $\Delta P / 2$ of $E_{p}$ and $P_{p}$, but may well lie on the neighboring array elements $j \pm 1$ and $k \pm 1$, relative to the position of $C_{94}$. The transit coefficient arrays from the individual years cannot, therefore, be directly added, since some planet candidates would thereby get lost.

An adding algorithm was therefore developed, which extends all local maxima of $C$ to their neighboring pixels in the yearly arrays, before adding the yearly arrays to an array describing the whole observations, that is $C_{94-98}$. This adding algorithm thus ensures - for a planet with parameters $\left(E_{p}, P_{p}\right)$ that would have caused a value of $C_{\max }$ in a very fine scan - that there will be a similar value, $C_{\text {alarm }}\left(E_{j}, P_{k}\right)$, in the whole observational array $C_{94-98}$ within a range of one array element of the intrinsic parameters $\left(E_{p}, P_{p}\right)$. This "neighborhood adding," used to obtain $C_{94-98}$, may, however, also promote unrelated events from the different years to cause local maxima in $C_{94-98}$. Values of $C_{\text {alarm }}\left(E_{j}, P_{k}\right)$ in $C_{94-98}$ are therefore only upper limits to a $C_{\max }$ that might be found with a much finer scan of the whole light curve.

Final high time resolution evaluation scans were therefore performed, testing the regions within a neighborhood of two array elements around all local maxima $C_{\text {alarm }}$, with a very fine grid (here we used $t_{j}=1526$ days and $f_{\mathrm{ov}}=0.5$ ), the results of which were $C_{\max }$, the final maxima found in the whole light curve. To find all planet candidates with $C_{\max }$ larger than some threshold $C_{\text {thres }}$, it was therefore necessary to scan $C_{94-98}$ in fine grids around all local maxima where $C_{\text {alarm }}>C_{\text {thres }}$. To find the best planet candidates, while ensuring that none were missed, it was necessary to scan fine grids around the 5000 best values of $C_{94-98}$. The resultant nine best candidates are given in $\S 5$ on detection results.

In order to determine a reasonable threshold $C_{\text {thres }}$ candidates found by the TDA, we turn now to a description of our TDA in terms of standard detection theory.

\section{THE MATCHED FILTER IN TERMS OF SIGNAL DETECTION THEORY}

The procedure for transit signal detection, as described in the previous section, is based on the matching of all possible planetary transit models against our differential light curve. The quality of these matches is numerically described by the complete transit statistic, $C$, which is our correlation statistic. The set of these transit statistics, resulting from testing against all possible planetary transit models, constitutes the statistic of detectability for a given size transiting planet in our observational light-curve data.

The detectability of a planetary transit signal can be pictured as the result of two hypotheses: $H_{0}$, the null hypothesis (no signals of planetary transits are present) and $H_{1}$, the detection hypothesis; that is, planetary transits are present in the light curve (see Jenkins et al. 1996, after Van Trees 1968). For the null hypothesis, $H_{0}$, we have used the set of all statistics $C(E, P)$, which were generated directly from the statistical test of our light-curve data against possible model transits, as described in the previous section. This set may contain one, but never more than very few, real planets. However, the number of real planets is, in any case, negligible when compared with the number of all possible distinguishable (with respect to the transit pattern they cause) planet transit models. We found the number of distinguishable planet transit models to be about $4 \times 10^{8}$ for our observational data coverage (see $\S 3$ ).

The $H_{1}$ hypothesis was generated by adding planetary transit models $M\left(E_{T}, P_{T}\right)$ to our data, and then obtaining the statistics $C\left(E_{T}, P_{T}\right)$ for these modified data (where $E_{T}$, $P_{T}$ indicate the parameters of the model planet). The set $C\left(E_{T}, P_{T}\right)$ for the $H_{1}$ hypothesis was calculated using 10,000 random values of $E_{T}$ and $P_{T}$.

Different $H_{0}$ and $H_{1}$ hypotheses can, of course, be generated for planets of different sizes, or for different ranges of periods, or from different subsamples of the CM Dra light curve. In all cases it is important, however, that the same assumptions are used for both the $H_{0}$ and $H_{1}$ hypotheses. In Figure 3 we show a histogram of the distribution of a null hypothesis $H_{0}$ and a detection hypothesis $H_{1}$. The horizontal axis is the detection statistic (the values of $C$ in the cases $H_{0}$ and $H_{1}$ ), while the vertical axis indicates the

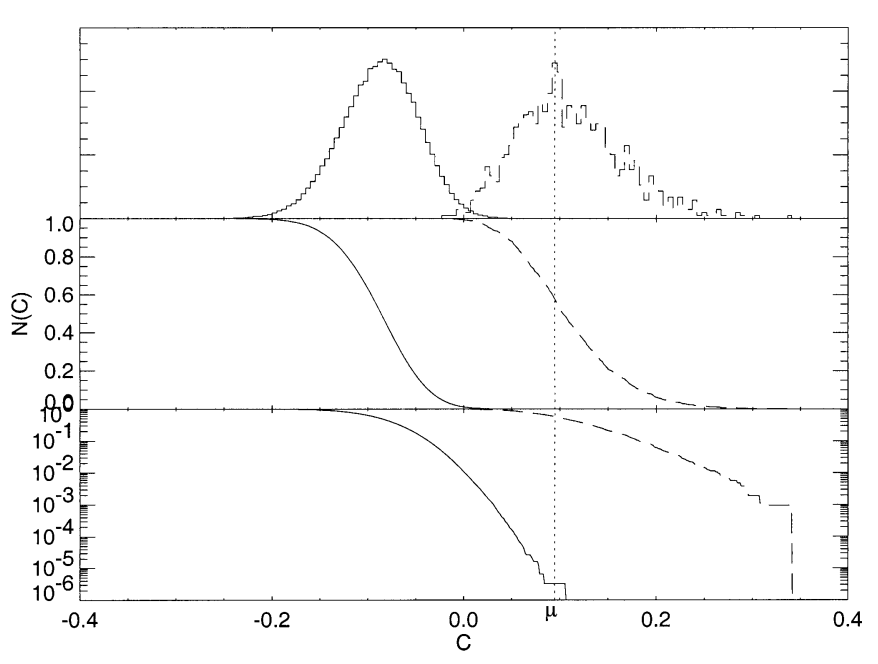

FIG. 3.-(Top) Sample $H_{0}-H_{1}$ plot showing the histogram (normalized to 1) of the distribution of the detection statistic given by the set of values $C$. The overlap of the planet-absent hypothesis, $H_{0}$ (left), with the planetpresent hypothesis, $H_{1}$ (right), determines the detectability. (In this example, of many possible examples, we show $H_{0}$ and $H_{1}$ diagrams from correlations against model planets of size $2.5 R_{\mathrm{E}}$, with periods between 7 and 10 days, which have caused seven transits in the light curve). (Middle) Same as the top panel, but $H_{0}$ and $H_{1}$ are plotted (starting at highest value) as cumulative histograms. (Bottom) Same as the middle plot but on a logarithmic scale that better shows the smallest values of $H_{0}$ and $H_{1}$. A threshold $\mu$ is set where the values of $H_{0}$ are smaller than $10^{-6}$ (dotted line). From the corresponding value $H_{1}(\mu) \geq 0.1$ in the middle panel one can see that the intrinsic detection probability $p_{i}$ of a planet corresponding to these distributions of $H_{0}$ and $H_{1}$ is about 0.55 . 


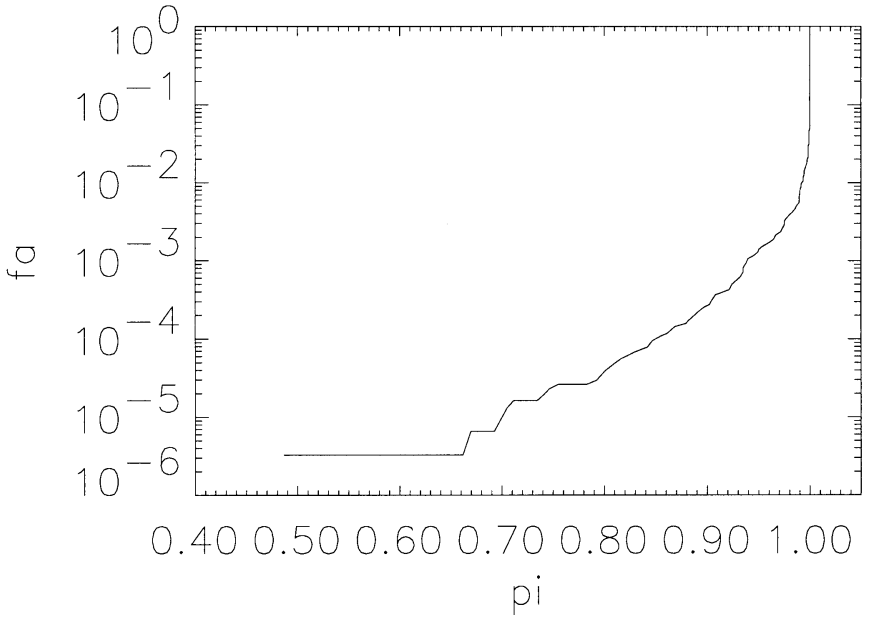

FIG. 4.-Relation between the intrinsic detection probability $p_{i}$ and the false alarm rate $f_{A}$. This figure is based on the same data set as in Fig. 3.

distribution of these values (normalized to 1 ). The separation of the $H_{0}$ and $H_{1}$ curves represents the detectability of, in this case, a planetary transit signal of a given size within the observational noise of the differential light curve. The separation of $H_{0}$ and $H_{1}$ will increase as the signal-to-noise ratio increases.

A threshold of detectability, $\mu$, can be selected in between the two hypotheses, and will determine the intrinsic detec-

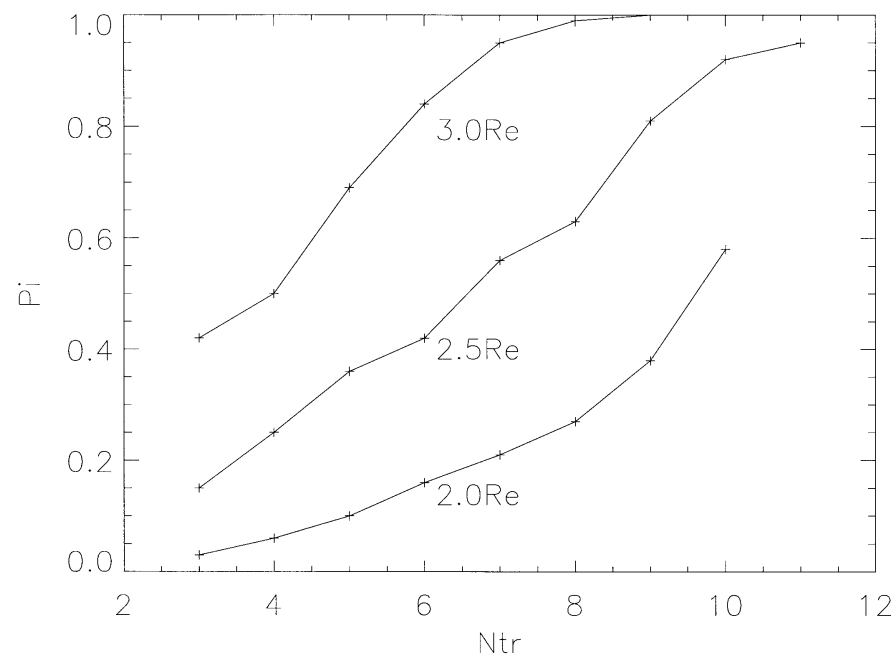

FIG. 6.-Values of $p_{i}=p_{i}\left(R, N_{\mathrm{tr}}\right)$ for $R=2.0,2.5$ and $3 R_{\mathrm{E}}$. All individual values have been derived from $H_{0}-H_{1}$ diagrams as shown in Fig. 4. Values of $p_{i}$ for $2.5 R_{\mathrm{E}}$ are based on the "complete" scan of $(E, P)$ parameter space $\left(5 \times 10^{7}\right.$ values of $\left.C\right)$, whereas the values of $p_{i}$ for 2.0 and $3.0 R_{\mathrm{E}}$ are based on "exploratory" scans through epoch-limited regions in $(E, P)$ parameter space, with $H_{0}$ distributions derived from about $4 \times 10^{5}$ values of $C$.

tion probability $p_{i}$, and the false alarm rate, $f_{A}$. The area to the left of $\mu$, yet still under the $H_{1}$ curve, is the probability of having missed a real planetary transit event that was in the data (i.e., $1-p_{i}$ ). The value of $1-p_{i}$ can be read off from the value of $H_{1}$ at $\mu$ in the reverse cumulative histogram,
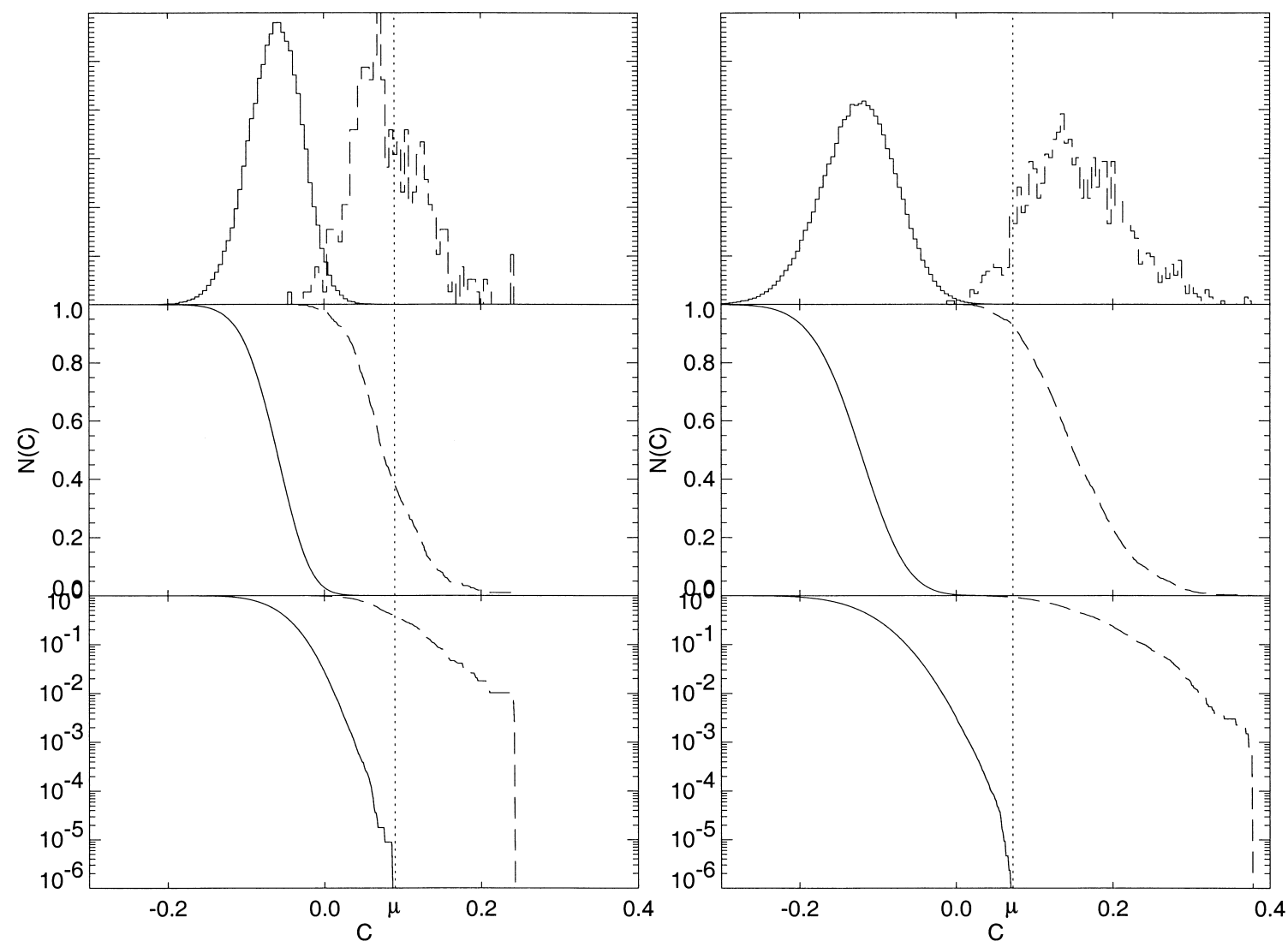

Fig. 5. $-H_{0}-H_{1}$ diagrams similar to Fig. 3 for a specific planet model with $2.5 R_{\mathrm{E}}$. Here the $H_{0}$ distribution is derived from about $5 \times 10^{7}$ values of $C$ calculated in the "complete" scan of $(E, P)$ parameter space (see $\S 3$ ). The $H_{1}$ distribution is obtained from the insertion of 10,000 test planets with random parameters $(E, P)$ into the light curve. From this large number of models, for the left-hand side those with $N_{\mathrm{tr}}=5$ have been selected, giving $p_{i} \approx 0.35 \pm 0.05$, and for the right-hand side those with $N_{\mathrm{tr}}=10$, giving $p_{i} \approx 0.92 \pm 0.03$. 


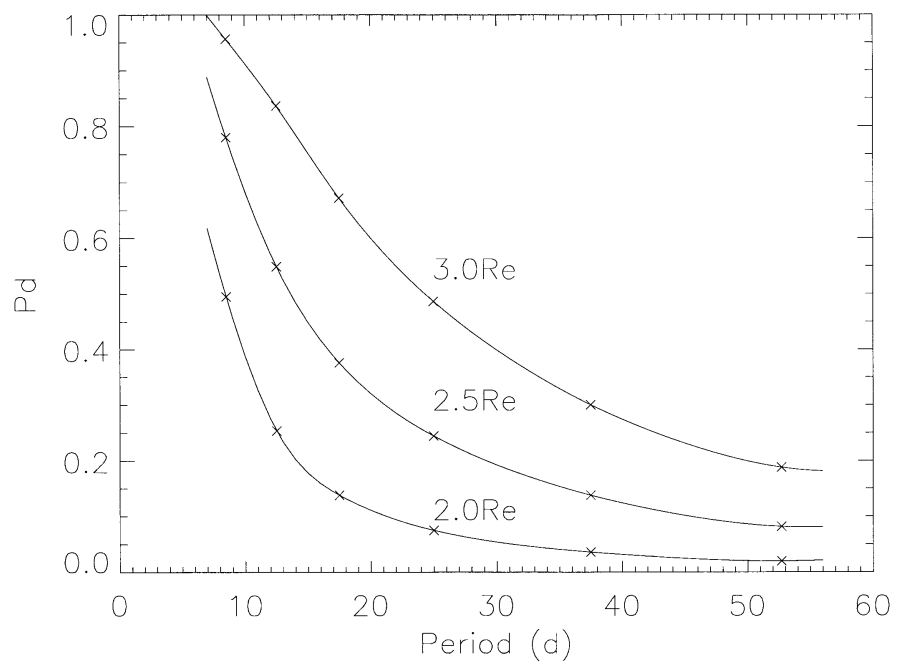

FIG. 7.-Values of $p_{d}(R, P)$ for $R=2.0,2.5$, and $3 R_{\mathrm{E}}$ obtained from the summation in eq. [13]. These values represent the final detection probabilities (a product of the photometric noise-limited precision, $p_{i}$, and the observational coverage, $p_{o}$ ) of planet candidates contained within the TEP light curve of CM Dra for the observational years 1994-1998.

which is an alternative way of plotting the detection hypothesis, $H_{1}(\mu)=p_{i}$ (Figs. $3 b$ and $3 c$ ). The goal of any signal detection scheme is, of course, not to miss much, while nevertheless simultaneously reducing the probability of false alarms to as low a value as possible.

To the right of the threshold $\mu$, where $H_{0}$ overlaps $H_{1}$, the null hypothesis has values that fall within the signalpresent area, i.e., within $H_{1}$. The ratio of the area of $H_{0}$ to the right of $\mu$, to the total area of $H_{0}$ (in Fig. 3a), defines the false-alarm rate $f_{A}$, which describes the ratio between the number of false alarms and all cases of $H_{0}$, that is, the probability that any arbitrary point in $(E, P)$ parameter space is a false alarm. In the cumulative histogram (Figs. 3, middle and bottom panels) therefore: $f_{A}=H_{0}(\mu)$. The relation between $p_{i}$ and $f_{A}$ is shown in Figure 4, (although it can also be read off from the middle and bottom panels of Fig. 3 and $3 c$ ). Since $H_{0}$ contains about $4 \times 10^{8}$ distinguishable cases in $(E, P)$ parameter space (see $\S 3$ ), $f_{A}$ needs to be exceedingly small to get the number of false alarms as small as possible (without missing any transit signals that could have shown up in the data). The value of $\mu$ was therefore chosen to produce a very small number of alarms, small enough to allow subsequent verification of the nature of each individual alarm observationally ( $\$ 5$, below). In the determinations of the intrinsic detection probability $p_{i}$ cited later, we set $\mu$ so that ${ }^{21} f_{A}=H_{0}(\mu) \approx 10^{-6}$, that is, our false-alarm rate was less than about 1 in 1 million.

The values of $C(E, P)$ that were included in the distributions of $H_{0}$ and $H_{1}$ had to fulfill a further requirement. This is that the number of transits, $N_{\mathrm{tr}}$, in the light curve that are needed to derive a planet candidate's parameters $E, P$ unambiguously is $N_{\mathrm{tr}}=3$. Requiring more transits increases the separation between $H_{0}$ and $H_{1}$ (Fig. 5), and thus

${ }^{21} H_{0}(\mu) \approx 10^{-6}$ can only be defined if the size of the sample is greater than about $10^{6}$. In the complete scan for $2.5 R_{\mathrm{E}}$ planets (mentioned in $\S 3$ and used for Figs. 3-5), the sample size is about $5 \times 10^{7}$. However, for many "exploratory" scans (such as for other planet sizes), the sample size was on the order of $10^{4}-10^{5}$. However, as can be seen in the bottom panel of Fig. 3, $\log H_{0}$ is fairly linear below $H_{0}=10^{-2}$, allowing a linear interpolation to $H_{0}=10^{-6}$. Values of $p_{i}$ derived this way do, however, have an error of about \pm 0.1 . increases $p_{i}$, but will simultaneously decrease $p_{o}$ by requiring more events (see Fig. 1). In the $H_{0}-H_{1}$ diagrams of Figure 3, the large numbers of models that create the $H_{0}$ and $H_{1}$ distributions have been separated by the number of transits $\left(N_{\text {tr }}\right)$ they contain, showing the strong dependency of $p_{i}$ on $N_{\text {tr }}$.

If $N_{\mathrm{tr}}$ is kept constant, the intrinsic detection probabilities $p_{i}$ depend strongly on the planet's size but are nearly independent of the planet's period. This is a consequence of the duration of the transits being only weakly dependent on the planetary period. Similarly, the observational detection probability $p_{o}$ varies strongly with the period, but is nearly independent of the planet's size, since the frequency of the transits is independent of the size, and the duration of the transits is affected in a minor way only, varying on the order of $\left(R+R_{*}\right) / R_{*}$, where $R_{*}$ is the star's radius. Therefore,

$$
p_{i}=p_{i}\left(R, N_{\mathrm{tr}}\right)
$$

(see Fig. 6) and

$$
p_{o}=p_{o}\left(P, N_{\mathrm{tr}}\right)
$$

(see Fig. $1 a$ ), i.e., $p_{i}$ is only a function of the number of transits and of the transiting planet's radius, while $p_{o}$ is only a function of the number of transits and of the planet's orbital period.

In a way similar to equation (1), which gives the total detection probability $p_{d}$ for a single candidate, the total detection probability for any candidate (which may appear with any value of $N_{\mathrm{tr}}$ ) can be obtained by:

$$
p_{d}(R, P)=\sum_{N_{\mathrm{tr}=3}}^{\infty} p_{i}\left(R, N_{\mathrm{tr}}\right) p_{o}\left(P, N_{\mathrm{tr}}\right) \text {. }
$$

The values of $p_{d}$, for planet sizes between 2 and $3 R_{\mathrm{E}}$, that were thus obtained from the analysis of our CM Dra light curve are shown in Figure 7.

\section{DETECTION RESULTS OF THE TDA ANALYSIS OF THE CM DRA LIGHT CURVE}

\subsection{Size Limits of the Detection Algorithm}

In TEP1 we preliminarily reported that no obvious transits for planets much larger than $2.5 R_{\mathrm{E}}$ had been seen in the light curve. However, they cannot be excluded entirely, as can be seen from the values of $p_{i}$ (Fig. 6). On the other hand, $2 R_{\mathrm{E}}$ turned out to be too optimistic a detection goal with the current data set size and precision. As can be seen in Figure 6, at least 10 transits are needed from a $2 R_{\mathrm{E}}$ planet to obtain over $50 \%$ detection probability within the current data. Using a "class 1" subset of about $35 \%$ of the full CM Dra light curve, consisting only of the best nights' data (requiring an $\mathrm{rms}$ of less than $0.4 \%$ ), values of $p_{i}$ for $2 R_{\mathrm{E}}$ similar to the ones reported for $2.5 R_{\mathrm{E}}$ with the full light curve were obtained. Unfortunately, with the coverage by "class 1 " data being much smaller $(340 \mathrm{hr})$, the observational detection probability $p_{o}$ is too low to maintain $2 R_{\mathrm{E}}$ as a realistic detection goal. An exception may be $2 R_{\mathrm{E}}$ planets with very short periods of less than 10 days, where detection probabilities approaching $50 \%$ could be obtained. For the complete TDA scan of the $(E, P)$ parameter space, which was computationally very intensive, we then choose planet models based on a size of $2.5 R_{\mathrm{E}}$. Since the TDA algorithm of $\S 3$ is sensitive to transits in the data caused by any planets larger than the $2.5 R_{\mathrm{E}}$ model planets, we thereby did not preclude the discovery of larger planets. (But, again, any transiting planet significantly larger than $3 R_{\mathrm{E}}$ should 
have been very obvious in the light curve, and its detection probabilities, which are governed by $p_{o}$ alone, would be very high (see Fig. $1 b$ for $N_{\mathrm{tr}, \min }=1$ ).

After constraining the optimum signal strength (i.e., planet size) to $2.5 R_{\mathrm{E}}$, then, the full scan through $E$ and $P$ for periods from 7 to 60 days was performed for such planets, (requiring a total of $5.6 \times 10^{7}$ planet models to be evaluated, as explained in $\S 3$ ). We note that the median precision of our photometry, about $0.45 \%$, corresponds to a single, central transit depth of a $2.6 R_{\mathrm{E}}$ planet around CM Dra, (that is, the TDA for a $2.5 R_{\mathrm{E}}$ planet has been operating at approximately the noise limit for one transit event). Because of the large, but still limited, data set as well as good, but limited, photometric precision, the TDA will not have been very sensitive to transits caused by planets smaller than about $2.5 R_{\mathrm{E}}$. For smaller planets, data with lower noise (such as the "class 1 " data mentioned above) and/or significantly longer observational coverage would be needed.

\subsection{Evaluation of Candidate Planets}

As a result of the search for maxima in the $C(E, P)$ array (see $\S 3$ ), nine top planetary transit candidates of various periods (but all with a preliminary size of $2.5 R_{\mathrm{E}}$ ), were determined from the data taken through 1998. The period of these candidate planets, their binary barycenter-crossing epochs, the value of the final transit coefficient, and the number of transit events in the light curve are given in Table 2 . These are the best transiting $2.5 R_{\mathrm{E}}$ planet models selected from more than 400 million possible period-epoch candidates. (A few further models were rejected, since they depended on one dominating attenuation event, or on events from only one observatory, both of which might be suspect.) Figures 8 and 9 show the attenuations in brightness in the light curve that have been fitted with the model transits for the 8.16 day candidate (the one with the highest number of transits) and for the 22.57 day candidate (the one that "survived" after the observations in summer 1999; see $\S 6)$. It is important to note that these candidate planetary transit events are clearly very close to the observational noise. In fact, there have to be top candidates in any such extensive search in the observational noise, and one would hope for one - or very few - clearly outstanding candidates. However, the complete transit statistics of these nine candidates were only about $20 \%$ above those of the next possible candidates. Given a field of over 400 million possible models, some distinguished, yet arbitrary, candidates may emerge. Hence the necessity of predicting and observationally confirming such candidates (see discussion $\S 5.3$ below).
As an additional note, when looking for transit events across eclipsing binaries, one must be aware of the binary orbital frequencies' contribution to photometric variability and thus of planetary orbital periods that are modulo the binary period. Candidates 3 and 4 in Table 2 (periods of 8.83 and 10.12 days) are within a few percentage points of being modulo CM Dra's period of 1.268 days. However, upon inspection, none of the photometric attenuation events that led to these candidates' high transit statistics shared any nearby sequential phase events with possible rotating features that might have been caused by photometric variations on the surface of CM Dra A or B. We note, however, that the periods of several of the planet candidates among themselves are related by multiples very close to integers (such as 8.83d:19.85d:26.44d 4:9:12); i.e., they could share several of the same transit events.

\subsection{Predicted Transit Detection Statistics and Observational Confirmation}

While the product of the probability of the transit being in our light curve $p_{o}$, with the signals of a given candidate having enough transit power ("energy" in signal detection nomenclature) to be detectable $p_{i}$, determines the total transit detectability, $p_{d}$, of a given candidate (eq. [1]), the confidence level in such candidates as being of a planetary transit nature can only be reliably estimated by the detection of a predicted transit event signal (i.e., a significant drop in stellar brightness "on schedule"). This detection confidence is due to the low probability that at the predicted time a transit signal, of the correct duration in the light curve, will be present that will increase the coefficient $C$ of the predicted individual candidate.

As previously stated, it is essential that the number of predicted candidates be kept relatively small. If a very large number of predicted candidates is to be verified, any attenuation in a future observed light curve (that is, any feature in the light curve that may lead to sufficiently high values of $\kappa$; see eq. [5]) could be assigned to at least one of very many candidates, thus invalidating the use of observational prediction as a tool to assess the reliability of any detection.

The probability that the prediction for a single candidate is valid can be estimated as follows. In our whole observational light curve, about $3 \%$ of the coverage data points cause positive values of $\kappa$ for transits lasting about 50 minutes (which is the most common kind of transit). Another way of stating this is that about 3\% of the light curve displays some feature (i.e., a series of points below the mean) that could be some kind of transit (remember that we are largely working at the mean photometric noise level). In

TABLE 2

Top Planetary Transit CANDidates from ObSERVATIONS IN 1994-1998 AND 1999

\begin{tabular}{cccccc}
\hline \hline $\begin{array}{c}P \\
\text { (days) }\end{array}$ & $E+$ JD 2,450,000 & $N_{\text {tr }}(1994-1998)$ & $C(1994-1998)$ & $N_{\text {tr }}(1999)$ & $C(1999)$ \\
\hline $7.67 \ldots \ldots$. & 6.86 & 8 & 0.109 & 1 & -0.014 \\
$8.16 \ldots \ldots$ & 2.15 & 11 & 0.129 & 3 & -0.039 \\
$8.83 \ldots \ldots$. & 8.81 & 8 & 0.129 & 2 & -0.014 \\
$10.12 \ldots \ldots$ & 5.35 & 5 & 0.144 & 1 & -0.033 \\
$12.03 \ldots \ldots$ & 8.08 & 9 & 0.149 & 2 & -0.012 \\
$19.85 \ldots \ldots$ & 14.56 & 5 & 0.129 & 2 & -0.017 \\
$20.56 \ldots \ldots$ & 19.94 & 7 & 0.129 & $\ldots$ & $\ldots$ \\
$22.57 \ldots \ldots$ & 13.77 & 6 & 0.116 & 1 & 0.004 \\
$26.44 \ldots \ldots$ & 2.31 & 5 & 0.124 & $\ldots$ & $\ldots$ \\
\hline
\end{tabular}



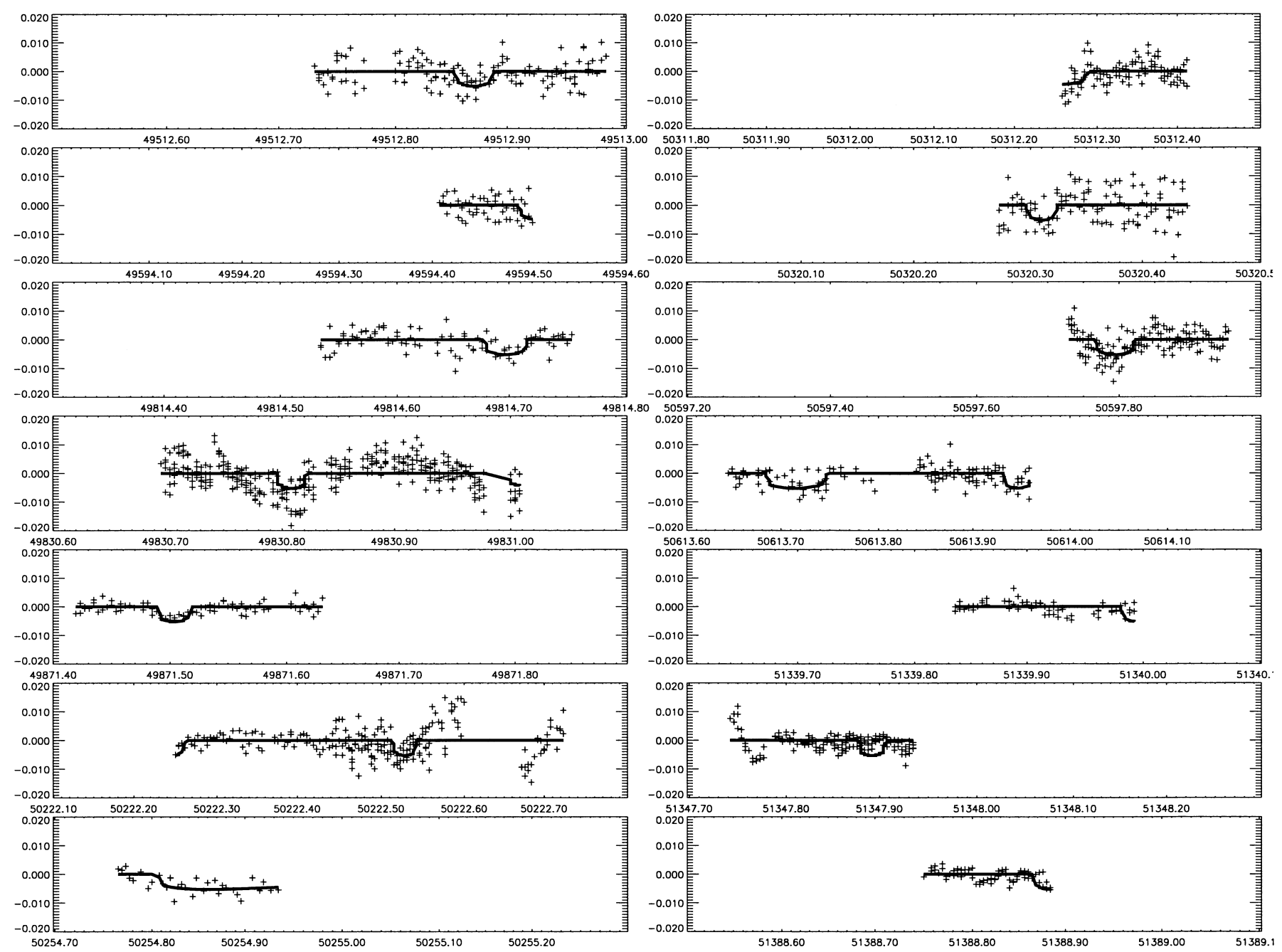

FIG. 8. - Each graph shows a barycenter crossing where the 8.16 day period candidate (solid line) would have caused transits in the observed light curve (crosses). The horizontal axis gives the time in Heliocentric Julian Days, the vertical one the brightness variation $\Delta F / F_{0}$. The 8.16 day candidate would have had the most transit events in the light curve between 1994 and 1998 (11 barycenter crossings; 14 whole or partial transit events altogether). In 1999 , observations at three nights to verify this candidate showed a negative result (last 3 graphs at lower right).

this, the nature of each transit feature is assumed to be unknown; it may be a transit from a planet but could also be due to observational photometric variability of any kind (as discussed in TEP1). The probability that, at a particular predicted time, a feature that may be a transit shows up is therefore about $3 \%$. This means that if such a feature is being observed at the predicted time, there is $97 \%$ confidence that this feature is related to (i.e., caused by) that predicted transit event-i.e., 1 minus the false-alarm rate for a random transit event. We note that the specific value of $3 \%$ holds, of course, only for photometric data of the approximate quality of the data obtained in our observations, and will, of course, be smaller for data with lower noise.

Since we looked for seven candidates 13 times $^{22}$ and found one possible surviving candidate out of 12 , our con-

\footnotetext{
${ }^{22}$ Seven candidates out of nine could be observed in 1999 (see Table 2) with three and two events searched for the 8.16 day and 12.0 day candidates, respectively. One 8.83 day period event corresponded to a 19.8 day candidate event, as well, but, for simplicity here, we will assume that all searches were essentially independent.
}

fidence in this candidate ${ }^{23}$ is 1 minus the probability that this could have happened randomly, that is: $1-(0.97)^{12}(0.03)(13)=73 \%$. Similarly, if the same planet candidate causes $n$ further observed transit events at the correctly predicted time, the probability of a false alarm is determined by

$$
p_{f_{A}}=(0.97)^{12}(0.03)(13)(0.03)^{n} .
$$

The probability of false alarm for additional observations is thus $0.81 \%, 0.024 \%, 0.00073 \%$, and so on, with the confidence in a detection increasing rapidly beyond $9.9 \%$ with additional predicted transit events of the 22.57 day candidate.

Clearly keeping the number of candidates that are to be tested by such predictions small is needed to validate these predictions. Fortunately, the predicted transit times for the candidates listed in Table 2 are almost entirely independent of each other in the sense that almost no predicted transit times for any candidate overlap. Thus, equation (14) will most closely apply to the estimation of the detection con-

${ }^{23}$ By keeping the factor of 13 we conservatively ignore the consideration that we know upon which trial the false alarm occurred. 

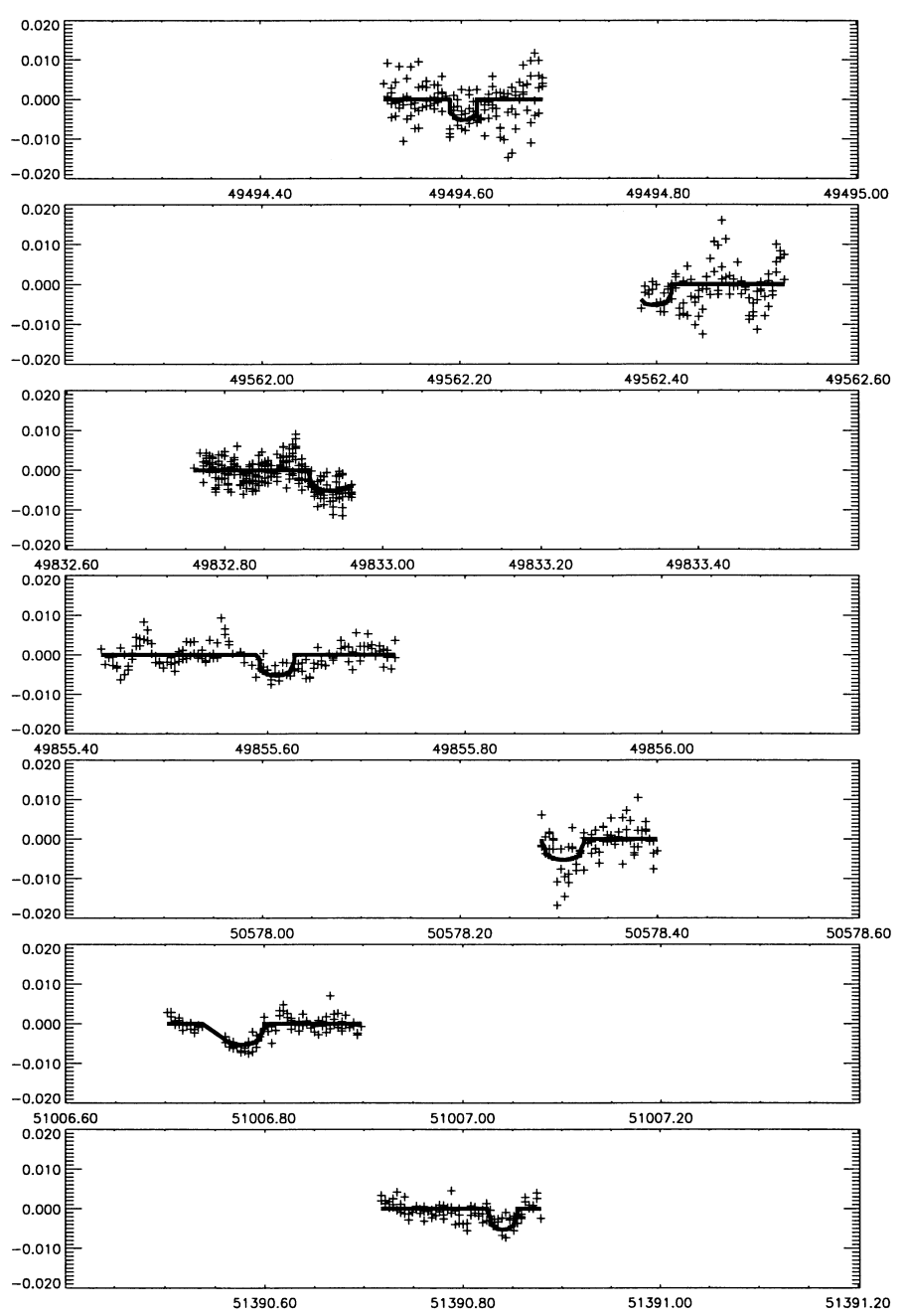

FIG. 9.- Like Fig. 8, with the model light curve for the 22.56 day period candidate, showing eight transit events. This candidate was the only one that displayed a positive predicted "transit" event in summer 1999 (last graph). Further (predicted) observations will thus be necessary to ascertain its validity as a planet or rule it out.

fidence resulting from our predicted transit events of the individual candidates given in Table 2.

\section{OBSERVATIONS OF PREDICTED CANDIDATE PLANETS}

\subsection{Observations}

Once specific candidate planets have been determined, specific follow-up observations based on the calculated ephemerides of transits can be performed. Ephemerides were calculated for the candidate planets listed in Table 2, and 12 predicted "transit" event times, belonging to seven of the nine candidates, were observed at Lick Observatory in the summer of 1999 . The 20.56 day and 26.44 day candidates could not be observed, owing to bad weather at the predicted times. For all of these observed planet candidates, resultant transit statistics were subsequently calculated for the observations in 1999. Results are included in Table 2, along with the number of predicted events covered and the transit statistics obtained in 1999.

In the case of the 8.16 day candidate-which had 11 previous suspected events - three predicted transit times were observed (Fig. 8). However, each of the three obser- vations resulted in a negative complete transit statistic, thus nullifying this candidate. This case may be the most extreme, but to some extent - with so many possible candidates (over 400 million searched near the noise level) - it can perhaps be understood that there is a nonvanishing probability that even good candidates may be consequences of random sequences of transit-like noise features. Again, this emphasizes the importance of predicted transit events and follow-up observations before a definitive small-planet detection (i.e., one near the observational noise) can be reliably claimed. Five more planet candidates were ruled out from the observations of one to three predicted transit times. Only the 22.57 day period candidate (Fig. 9) showed a positive transit statistic from the one predicted transit that was observed. In summary, in this work to date, we have taken the highest nine candidates isolated from other possible planets in epoch and phase by the TDA. However, in the case of CM Dra, the resultant candidates were neither very far above the observational noise nor significantly (about 20\%) above many other candidates. Nevertheless, the "surviving" candidate (with the 22.57 day period transit signature) will need to be observationally confirmed or ruled out to be definitive. As discussed in $\S 5.3$ above, the confidence in such a candidate should grow as equation (14) indicates, with the specific percentage of $3 \%$ being dictated by the photometric noise of the observational program. Observational confirmation of the 22.57 day candidate will therefore be a high priority.

\section{DISCUSSION}

The binary nature of our target stellar system introduces several complications for planetary transit detections when compared to the detection of planetary transits around single stars. For example, we had to remove the mutual binary eclipses and also calculate, for a specific model, $M(E, P)$, each configuration of $E$ and $P$. Beyond these differences, however, the method outlined here should be applicable to detecting transits around most kinds of stellar systems. In the case of a planet transiting a single star, a model, $M=M(P, i)$, can be calculated only with reference to the period (and the inclination, $i$, if desired), where $P$ only weakly affects the duration of the transit, but not its shape, and $i$ affects mainly the amplitude. Independent of the details of how the transit statistic $C$ is precisely derived, the determination of the detection probability and the falsealarm rate using the $H_{0}$ and $H_{1}$ hypothesis can be applied. We expect that even for space-based transit observations (which would have observational coverage of much higher uniformity) an analysis of the dependence on the number of transits $\left(N_{\text {tr }}\right)$ of the model planets, similar to the one outlined here, will also have to be performed.

Detection of photometric variations due to transits is presently the only repeatable method for the investigation of possible extrasolar planets around close binaries that we are aware of - the binary nature of these systems making them, at present, too complex to study for minute radial velocity variations, for example. However, in the photometric case, the quasi-periodicity of the expected variation actually helps to establish a more unique series of transit signatures, generally allowing one to rule out more periodic events of intrinsic stellar variability. In addition, for ground-based observations, binary transit events can generally be expected to contribute their power at higher frequencies (50 minute transits vs. several hour-long transits) 
and thereby to be more easily separable from the higher amplitude low-frequency observational noise (see Doyle et al. 1996, TEP1).

At present there seem to be no compelling reasons for arbitrarily excluding close binary systems as sites of planet formation. Stability considerations (Donnison \& Mulkulskis 1992; Holman \& Wiegert 1999) as well as discoveries of circumstellar material (Jensen, Koerner, \& Mathieu 1996; Kalas \& Jewett 1997, for example) rather indicate that close binaries could be active sites of planet formation. With the discovery of a Jovian-mass planet around Gliese 876 (Marcy et al. 1998) clearly there also seems to be nothing intrinsic to $\mathrm{M}$ stars that would exclude planet formation around them (although stellar populations of differing metallicities could easily be seen to produce significant differences in planetary formation rates). Recent atmospheric models, as well, have not excluded smaller, inner M star planets as potential sites of liquid water (Haberle et al. 1996; Joshi, Haberle, \& Reynolds 1997; Heath et al. 1999).

\section{CONCLUSIONS}

We have performed a search for terrestrial-sized planets around another main-sequence star system, reaching a planet detection limit size of $2.5 R_{\mathrm{E}}$ (i.e., about $1 \%$ the volume of Jupiter). While we have observed one of the smallest known eclipsing binaries, CM Dra, we have also used only 1 meter class ground-based telescopes. Clearly this method may be extended to more crowded stellar fields (many simultaneous targets; Doyle et al. 2000) as well as to larger stellar systems. In the case of larger eclipsing binary systems, one may expect the photometric precision to improve both with the square root of the light-gathering power of the telescope and with the significantly decreased scintillation noise that can be expected from larger telescopes (Dravins et al. 1998, for example).

Whether the current planet candidate turns out to be a true detection or simply a photometric noise artifact, the methodology outlined herein has hopefully demonstrated the validity of pursuing the detection of small inner (i.e., terrestrial-sized) planets around late-type main-sequence stars using this transit/matching algorithm technique and existing observational facilities. This method should find application to near-term spacecraft missions, as well. This may be the best approach to at least begin to answer that most intriguing of planetary detection questions: "Are there other habitable planets?"

L. R. D. wishes to thank the SETI Institute for a special observing grant, and the University of California, Lick Observatory, allocation committee for continued substantial time on the Crossley telescope (incidentally, the oldest professional-sized reflecting telescope, first silvered in the late 1870s; see Stone 1979). H. J. D. acknowledges a grant "Formación de Personal Investigador" by the Spanish Ministerio de Educación y Cultura for the years 1997 and 1998. The IAC80 and European Space Agency OGS telescopes are operated at Izaña Observatory, Tenerife, by the Instituto de Astrofísica de Canarias. Capilla Peak Observatory is operated by the Institute for Astrophysics of the University of New Mexico. (The contributions of the other previously participating observatories for the years 1994 1996 are given in TEP1.)
Borucki, W. J., \& Summers, A. L. 1984, Icarus, 58, 121

Brandmeier, S., \& Doyle, L. R. 1996, in Proc. First Int. Conf. on Circumstellar Habitable Zones, ed. L. R. Doyle (Menlo Park: Travis House), 251

Deeg, H.-J. 1998, in ASP Conf. Ser. 134, Brown Dwarfs and Extrasolar Planets, ed. R. Rebolo, E. L. Martin, \& M. R. Zapaterio-Osorio (San Francisco: ASP), 216

Deeg, H. J., et al. 1998, A\&A, 338, 479 (TEP1)

Deeg, H. J., \& Doyle, L. R. 2000, in Proc. Extrasolar Planets Photometry Workshop, ed. W. Borucki \& D. Koch (NASA Ames Research Center Special Publication), in press

Deeg, H. J., Doyle, L. R., Kozhevnikov, V., Blue, J. E., Martin, E. L., \& Schneider, J. 2000, A\&A Lett., submitted

Deeg, H. J., Martin, E. L., Doyle, L., Jenkins, J., Schneider, J., Chevreton, M., \& Palaiologou, M. 1997, A\&A Trans., 13, 233

Donnison, J. R., \& Milkulskis, D. F. 1992, MNRAS, 254, 21

Doyle, L. R., et al. 1998, in ASP Conf. Ser. 134, Brown Dwarfs and Extrasolar Planets, ed. R. Rebolo, E. L. Martin, \& M. R. Zapaterio-Osorio (San Francisco: ASP), 224

Doyle, L. R., Dunham, E. T., Deeg, H. J., Blue, J. E., \& Jenkins, J. M. 1996, J. Geophy. Res. (Planets), 101, 14,823

Doyle, L. R., Deeg, H. J., Blue, J. E., Navaarate, M., \& Rottler, L. 2000, in Bioastronomy: A New Era in the Search for Life in the Universe, ed. G. A. Lemarchand (San Francisco: ASP), in press

Dravins, D., Lindegren, L., Mezey, E., \& Young, A. T. 1998, PASP, 110, 610

\section{EFERENCES}

Gilliland, R. L., \& Brown, T. M. 1992, PASP, 104, 582

Haberle, R. M., McKay, C. P., Tyler, D., \& Reynolds, R. T. 1996, in Proc. First Int. Conf. on Circumstellar Habitable Zones, ed. L. R. Doyle (Menlo Park: Travis House), 29

Hale, A., \& Doyle, L. R. 1994, Ap\&SS, 212, 51

Heath, M., Doyle, L. R., Joshi, M., \& Haberle, R. M. 1997, Origins of Life, 29,405

Hertz, P., Wood, K. S., \& Cominsky, L. 1995, ApJ, 438, 385

Holman, M. J., \& Wiegert, P. A. 1999, AJ, 117, 621

Jenkins, J. M., Doyle, L. R., \& Cullers, D. K. 1996, Icarus, 119, 244

Jensen, E. L. N., Koerner, D. W., \& Mathieu, R. D. 1996, AJ, 111, 2431

Joshi, M. M., Haberle, R., \& Reynolds, R. T. 1997, Icarus, 129, 450

Kalas, P., \& Jewitt, D. 1997, Nature, 386, 52

Lacy, C. H. 1977, ApJ, 218, 444

Marcy, G. W., Butler, R. P., Vogt, S. S., Fischer, D., \& Lissauer, J. 1998, ApJ, 505, 147

Rosenblatt, F. 1971, Icarus, 14, 71

Schneider, J. 1994, Planet. Space Sci., 42, 539

Schneider, J., \& Chevreton, M. 1990, A\&A, 232, 251

Schneider, J., \& Doyle, L. R. 1995, Earth Moon Planets, 71, 153

Stone, R. P. S. 1979, S\&T, 58, 307

Struve, O. 1951, Observatory, 72, 199

Van Trees, H. L. 1968, Detection, Estimation, and Modulation Theory, Part I (New York: Wiley)

Young, A., et al. 1991, PASP, 103, 221 